\title{
Long term outcome of prophylaxis for febrile convulsions
}

\author{
F U Knudsen, A Paerregaard, R Andersen, J Andresen
}

\begin{abstract}
A cohort of 289 children with febrile convulsions who had been randomised in early childhood to either intermittent prophylaxis (diazepam at fever) or no prophylaxis (diazepam at seizures) was followed up 12 years later. The study focused on the occurrence of epilepsy and on neurological, motor, intellectual, cognitive, and scholastic achievements in the cohort.

At follow up the two groups were of almost identical age $(14.0 v 14.1$ years), body weight $(58.2 v 57.2 \mathrm{~kg})$, height $(168 \cdot 2$ $v 167.7 \mathrm{~cm})$, and head circumference $(55.9$ $v 56.2 \mathrm{~cm})$. The occurrence of epilepsy $(0.7 \% v 0.8 \%)$, neurological examination, fine and gross motor development on the Stott motor test, intellectual performance on the Wechsler intelligence scale for children verbal IQ (105 $v$ 105), performance IQ (114 v 111), and full scale IQ (110 $v$ 108), cognitive abilities on a neuropsychological test battery, including short and long term, auditory and visual memory, visuomotor tempo, computer reaction time, reading test, and scholastic achievement were also very similar. Children with simple and complex febrile convulsions had the same benign outcome. The long term prognosis in terms of subsequent epilepsy, neurological, motor, intellectual, cognitive, and scholastic ability was not influenced by the type of treatment applied in early childhood. Preventing new febrile convulsions appears no better in the long run than abbreviating them.

(Arch Dis Child 1996; 74: 13-18)
\end{abstract}

Keywords: febrile convulsions, prophylaxis, long term prognosis.

Recent epidemiological data from major cohort studies document a normal long term outcome for most children with febrile convulsions ${ }^{1-7}$ and continuous prophylaxis for years with antiepileptic agents has largely been abandoned..$^{8-11}$ However, it is still unclear whether febrile convulsions may be associated with an adverse outcome in various, more subtle aspects of motor, neurological, intellectual, or cognitive functions, ${ }^{12} 13$ and whether medical intervention in early childhood has any impact on the long term prognosis, including the occurrence of subsequent epilepsy. These problems have not been studied in a randomised, controlled design with a long term follow up.

We report a long term follow up of the occurrence of epilepsy, and of neurological, motor, cognitive, and scholastic achievement in a well defined, historical cohort of children, ${ }^{14} 15$ with a mean age of 14 years, who in early infancy had been randomised to prophylaxis (diazepam at fever) or no prophylaxis (diazepam at ongoing seizures) after their first febrile convulsion. The baseline clinical and demographic characteristics of the two groups were comparable. ${ }^{14}{ }^{15}$ However, the 18 month recurrence rate was significantly lower in the prophylaxis group $(12 \% v 38 \%) .{ }^{14}$

\section{Methods}

THE COHORT

The children were admitted to our paediatric department with their first simple or complex febrile convulsion between June 1978 and June 1980 and assigned consecutively to prophylaxis or to be controls in a prospective, two branched, randomised design. Children admitted on even dates $(n=152)$ were given prophylaxis at home by the parents during future febrile episodes with rectal diazepam in solution 5-7.5 mg every 12 hours, whenever the rectal temperature was above $38.5^{\circ} \mathrm{C}$. A maximum of four consecutive doses were given during a febrile episode. Those admitted on odd dates $(n=137)$ were given rectal diazepam in solution in similar doses, but only in case of further febrile convulsions and no prophylaxis was given. The children were seen for outpatient follow up at three, six, 12 , and 18 months and number and types of new febrile and non-febrile convulsions were recorded (for details see Knudsen $\left.{ }^{14}{ }^{15}\right)$. The following entry criteria were met: (1) local residence in a well defined Copenhagen suburb area, (2) a first simple or complex febrile convulsion, regardless of age, and (3) no history of prior afebrile convulsions, overt chronic neurological disorder including severe psychomotor retardation, or purulent meningitis at the time of the febrile convulsion. Data on pregnancy, birth, birth weight, sex, age at the first febrile convulsion, febrile convulsions in parents and siblings, epilepsy in parents and siblings, type of first and later febrile convulsions, child care, and psychomotor development at the time of the first febrile convulsion were recorded. An electroencephalogram (EEG) was obtained one month after the first attack.

A febrile convulsion or seizure was defined as an event in infancy or childhood associated with fever at or above $38^{\circ} \mathrm{C}$ (rectal temperature) but without evidence of intracranial infection or defined cause.

Simple febrile convulsions were defined as generalised convulsions, lasting less than 15 minutes, not recurring within 24 hours and 
Table 1 Neuropsychological test battery

\begin{tabular}{|c|c|}
\hline Test & Functions assessed \\
\hline \multicolumn{2}{|l|}{ General tests } \\
\hline \multicolumn{2}{|l|}{$\begin{array}{l}\text { Specific ability tests } \\
\text { Memory }\end{array}$} \\
\hline $\begin{array}{l}\text { Memory } \\
\text { Logic memory (Binet: the Goldsmith }\end{array}$ & \\
\hline $\begin{array}{l}\text { Logic memory (Binet: the Goldsmith } \\
\text { story) }\end{array}$ & $\begin{array}{l}\text { Comprenension and wording. Short term auditive } \\
\text { memory, } 1 \text { hour retention of meaningful material }\end{array}$ \\
\hline Visual, digit memory (digit span) & $\begin{array}{l}\text { Visual recognition and recall of digits, forward and } \\
\text { backward }\end{array}$ \\
\hline Auditive, verbal memory free recall & Short term auditive memory, 10 minutes retention \\
\hline Non-verbal memory $\left(\right.$ BVRT $\left.^{\star}\right)$ & $\begin{array}{l}\text { Non-verbal short term memory, } 10 \text { minutes } \\
\text { retention, visuomotor ability }\end{array}$ \\
\hline $\begin{array}{l}\text { Visuomotor speed } \\
\text { Trail A and B }\end{array}$ & Visuomotor ability, visual search \\
\hline $\begin{array}{l}\text { Continuous visual reaction time test } \\
\text { (computer test) }\end{array}$ & $\begin{array}{l}\text { Attention, vigilance, visuomotor coordination, } \\
\text { visuomotor speed }\end{array}$ \\
\hline Reading test (standardised Danish text) & Reading rate, number of errors \\
\hline
\end{tabular}

${ }^{\star} \mathrm{BVRT}=$ Benton's revised visual retention test.

without any postictal neurological abnormalities. Complex febrile convulsions were defined as focal, long lasting, recurring within 24 hours, or associated with postictal neurological abnormalities.

\section{FOLLOW UP}

The follow up focused on the occurrence of epilepsy, neurological assessment, motor development, neuropsychological testing, school achievement assessment (teacher questionnaire), and a questionnaire to the parents and children. The cohort $(n=289)$ was approached by explanatory letters to parents and children and an invitation to take part in the study; this comprised a questionnaire to the family, a visit to the hospital for neurological, motor, and neuropsychological assessment, and an evaluation of the child's achievement in school, as evaluated by a questionnaire to the teacher.

\section{EPILEPSY}

Classification of convulsion type, type of epilepsy, and epileptic syndrome was done according to the International League Against Epilepsy's International Classification from 1981 and 1989 and was based on a full record and serial EEGs, and a long term follow up in our epilepsy clinic. Epilepsy was defined as two or more afebrile seizures.

NEUROLOGICAL ASSESSMENT

A standardised neurological examination was made by two of us (FUK and AP) comprising 12 items: general appearance, eyes, walking, running, walking on heels, toes, inner and outer foot edge, diadochochinesis, finger opposition, hand opposition, reflexes, tonus, trophic, and force. A score from 0-12 measured the number of abnormal responses. The assessment and scoring was made before the child's previous treatment status was discussed.

\section{MOTOR DEVELOPMENT}

This was evaluated by the Stott motor test, ${ }^{16}$ which in our slightly modified form contained a battery of 10 short tests involving gross motor control (one foot toe balance, two board balance, board balance 1 and 2, ring and coathanger, hitting target, catching one hand, jumping within circles, sideways hopping and jump with one foot landing) and four tests on fine motor control (track rotating, piercing holes, simultaneous piercing, and simultaneous pegs and squares). Each subtest scored 0-2 points, and each child scored 0-28 points, 0 being the best performance.

\section{NEUROPSYCHOLOGICAL ASSESSMENT}

Selection of the psychological tests employed was governed by the wish to cover a wide range of cognitive functions (table 1 and the appendix). The test battery consisted of a general intelligence test: Wechsler intelligence scale for children (WISC) abbreviated form (subtests included: information, similarities, arithmetic, vocabulary, digit span, picture arrangement, block design, coding) and a group of more specific neuropsychological tests. The functions assessed by the neuropsychological tests were attentional, visuomotor, and short and long term memory for the auditory and visual modalities. The neuropsychological tests are described in details in the appendix. A standardised Danish reading test scored for number of errors and reading time was also included. All tests were selected by the neuropsychologist (JA). They were carried out and scored by the same psychologist (RA) without any prior knowledge of the children's previous treatment status.

\section{SCHOLASTIC ACHIEVEMENT ASSESSMENT}

(TEACHER QUESTIONNAIRE)

A subjective, semiquantitative measurement of the child's scholastic ability was obtained by asking the child's teacher to evaluate if the child's current level of ability was above (2 points), average ( 1 point), or below ( 0 point) mean performance for their grade in spoken Danish, written Danish, mathematics, and physical education. Total obtainable score on the scale was 8 points. The teacher was unaware of the child's previous treatment status.

\section{QUESTIONNAIRE TO THE PARENTS}

Parents and children completed a questionnaire about prior or present epilepsy, single afebrile convulsions, faintings, migraine, tension-type headache, and a history of febrile or afebrile convulsions among first degree relatives. We also asked about reading or writing problems, clumsiness, physical noncompetitiveness, and referral for special teaching.

DATA COLIECTION, STATISTICS, AND ETHICS Data collection and testing took place between April 1991 and August 1992. At the time of follow up two children had died for reasons unrelated to febrile convulsions, seven had emigrated, eight others were not reached 
Table 2 Epilepsy subsequent to febrile convulsions

\begin{tabular}{|c|c|c|c|c|c|c|}
\hline \multirow[b]{2}{*}{ Epilepsy } & \multirow{2}{*}{$\begin{array}{l}\text { No of } \\
\text { children }\end{array}$} & \multicolumn{2}{|c|}{ Febrile convulsion } & \multirow{2}{*}{$\begin{array}{l}\text { Severity } \\
\text { of } \\
\text { epilepsy }\end{array}$} & \multirow{2}{*}{$\begin{array}{l}\text { Epilepsy } \\
\text { at } \\
\text { follow up }\end{array}$} & \multirow{2}{*}{$\begin{array}{l}\text { Scholastic } \\
\text { achievemen } \\
(0-8) \text { at } \\
\text { follow up }\end{array}$} \\
\hline & & $T y p e^{\star}$ & No & & & \\
\hline Rolandic epilepsy & 1 & $\mathrm{~s}$ & 3 & Mild & No & 7 \\
\hline Juvenile myoclonic epilepsy & 1 & s & 1 & Severe & Yes & 7 \\
\hline Epilepsy with tonic-clonic seizures & 1 & $\mathrm{C}$ & 3 & Mild & No & 3 \\
\hline Epilepsy with tonic-clonic seizures & 1 & & 4 & Mild & No & 4 \\
\hline Absence epilepsy & 1 & 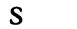 & 1 & Mild & No & 4 \\
\hline Absence epilepsy & 1 & $\mathrm{C}$ & 12 & Average & Yes & 2 \\
\hline
\end{tabular}

$\star \mathrm{S}=$ simple; $\mathrm{C}=$ complex.

despite several attempts, leaving 272 children. The questionnaire to the parents and children was returned in 268 cases (93.4\%), information about scholastic attainment, as assessed by the teacher, in 247 cases $(86.1 \%)$, and a total of 166 children $(57 \cdot 8 \%)$ accepted a full neurological, motor, intellectual, and cognitive assessment. No significant differences in teacher evaluation of scholastic abilities $(4.51 \mathrm{v}$ 4.34; not significant) and mean number of febrile convulsions $(2 \cdot 26 v 2 \cdot 26$; not significant) were found between the 268 children, where information from the parents was available, and the 166 children who agreed to participate in the medical and neuropsychological assessment, making selection bias less likely. However, the group subjected to neuropsychological testing was four months younger than the untested group ( $13.9 v 14.3$ years; $p=0.01)$ and girls outnumbered boys $(68.5 \%$ v $56.4 \%$; $\mathrm{p}=0 \cdot 04$ ).

Pearson $\chi^{2}$ and Kruskal-Wallis tests were used in the statistical evaluation. The final statistical analysis reached similar overall results whether performed with or without the exclusion of six children with neurological disorders, seemingly unrelated to the febrile convulsion (preterm children with a birth weight less than $1500 \mathrm{~g}$ suffering from neurological sequelae related to perinatal hypoxic-ischaemic encephalopathy $(n=3)$, hemiplegia after a purulent meningitis not associated with a febrile convulsion $(n=1)$, Gilles de la Tourette disorder $(n=1)$, and Charcot-Marie-Tooth disorder $(n=1))$. Informed consent was obtained and the study was approved by the local research ethics committee.

\section{Results}

Six of 272 children $(2 \cdot 2 \%)$ suffered from subsequent epilepsy at some point (table 2 ). At the time of follow up only two children had epilepsy $(0 \cdot 7 \%)$. The six cases were classified as idiopathic generalised epilepsy $(n=5)$ and idiopathic partial epilepsy $(n=1)$. Not a single case of temporal lobe epilepsy was seen (table 2). Five cases were mild, four disappeared within two years, and none were intractable. The risk of having epilepsy at the mean age of 14 years was one in $250(0.4 \%)$ after simple febrile convulsions and six times higher after complex febrile convulsions (one in $40,2.5 \%$ ); this was not a significant difference. The risk of having epilepsy associated with poor scholastic achievement (school score $\leqslant 2$ ) was one in 272 $(0.4 \%)$. The long term incidence of subsequent epilepsy was uninfluenced by prior prophylaxis $(0 \cdot 8 \%)$ compared with acute anticonvulsant treatment only $(0.7 \%)$, emphasising that prophylaxis did not offer any or any better protection against the rare cases of later epilepsy, compared with acute anticonvulsant treatment.

Mean age, body weight, height, and head circumference were almost identical for the prophylaxis and the control group (table 3 ). The neurological examination was normal in the vast majority of cases and no significant differences between the two groups were seen either for the neurological examination or for the fine and gross motor function test (table 4).

Table 3 Long term outcome after prophylaxis or no prophylaxis; physical data

\begin{tabular}{|c|c|c|c|c|c|c|c|c|}
\hline & \multicolumn{2}{|c|}{ Prophylaxis } & \multicolumn{2}{|l|}{ Control } & \multicolumn{2}{|l|}{ Total } & \multirow[b]{2}{*}{$\begin{array}{l}95 \% \text { CI for } \\
\text { difference }\end{array}$} & \multirow[b]{2}{*}{$\stackrel{p}{\text { Value }}$} \\
\hline & $\begin{array}{l}\text { No of } \\
\text { children }\end{array}$ & Mean (SD) & $\begin{array}{l}\text { No of } \\
\text { children }\end{array}$ & Mean (SD) & $\begin{array}{l}\text { No of } \\
\text { children }\end{array}$ & Mean (SD) & & \\
\hline $\begin{array}{l}\text { Age (years) } \\
\text { Height }(\mathrm{cm}) \\
\text { Body weight }(\mathrm{kg}) \\
\text { Head circumference }(\mathrm{cm})\end{array}$ & $\begin{array}{r}137 \\
91 \\
90 \\
90\end{array}$ & $\begin{array}{c}14 \cdot 0(1 \cdot 2) \\
168 \cdot 2(9 \cdot 0) \\
58 \cdot 2(14 \cdot 1) \\
55 \cdot 9(2 \cdot 2)\end{array}$ & $\begin{array}{r}128 \\
76 \\
76 \\
75\end{array}$ & $\begin{array}{c}14 \cdot 1(1 \cdot 3) \\
167 \cdot 7(8 \cdot 2) \\
57 \cdot 2(11 \cdot 6) \\
56 \cdot 2(1 \cdot 9)\end{array}$ & $\begin{array}{l}265 \\
167 \\
166 \\
165\end{array}$ & $\begin{array}{c}14 \cdot 1(1 \cdot 3) \\
167 \cdot 9(8 \cdot 7) \\
57 \cdot 8(13 \cdot 0) \\
56 \cdot 0(2 \cdot 0)\end{array}$ & $\begin{array}{l}-0.22 \text { to } 0.40 \\
-3.49 \text { to } 1.91 \\
-5.67 \text { to } 2.47 \\
-0.58 \text { to } 0.70\end{array}$ & $\begin{array}{l}\text { NS } \\
\text { NS } \\
\text { NS } \\
\text { NS }\end{array}$ \\
\hline
\end{tabular}

$\mathrm{CI}=$ confidence interval; $\mathrm{NS}=$ not significant.

Table 4 Long term outcome after prophylaxis or no prophylaxis; neurological, motor, intellectual, and scholastic ability

\begin{tabular}{|c|c|c|c|c|c|c|c|}
\hline & \multicolumn{2}{|c|}{ Prophylaxis } & \multicolumn{2}{|l|}{ Control } & \multicolumn{2}{|l|}{ Total } & \multirow[b]{2}{*}{$\stackrel{p}{\text { Value }}$} \\
\hline & $\begin{array}{l}\text { No of } \\
\text { children }\end{array}$ & Mean (SD) & $\begin{array}{l}\text { No of } \\
\text { children }\end{array}$ & Mean (SD) & $\begin{array}{l}\text { No of } \\
\text { children }\end{array}$ & Mean (SD) & \\
\hline $\begin{array}{l}\text { Neurological examination } \\
\text { Stott motor test }\end{array}$ & $\begin{array}{l}87 \\
86\end{array}$ & $0.51(1.4)$ & $\begin{array}{l}74 \\
72\end{array}$ & $0.71(1.61)$ & $\begin{array}{l}161 \\
158\end{array}$ & $0.60(1.5)$ & NS \\
\hline $\begin{array}{l}\text { Fine motor } \\
\text { Gross motor } \\
\text { Total motor }\end{array}$ & & $\begin{array}{l}1 \cdot 3(1 \cdot 5) \\
3 \cdot 0(3 \cdot 3) \\
4 \cdot 4(4 \cdot 4)\end{array}$ & & $\begin{array}{l}1.6(1.6) \\
3.4(3.5) \\
5 \cdot 2(4.9)\end{array}$ & & $\begin{array}{l}1 \cdot 4(1 \cdot 6) \\
3 \cdot 2(3 \cdot 4) \\
4 \cdot 8(4 \cdot 6)\end{array}$ & $\begin{array}{l}\text { NS } \\
- \\
-\end{array}$ \\
\hline WISC & 87 & & 74 & & 161 & & \\
\hline $\begin{array}{l}\text { Verbal IQ } \\
\text { Performance IQ } \\
\text { Full scale IQ }\end{array}$ & & $\begin{array}{l}105(16) \\
114(14) \\
110(14)\end{array}$ & & $\begin{array}{l}105(16) \\
111(19) \\
108(15)\end{array}$ & & $\begin{array}{l}105(16) \\
112(17) \\
109(15)\end{array}$ & $\begin{array}{l}\text { NS } \\
- \\
-\end{array}$ \\
\hline $\begin{array}{l}\text { Scholastic achievement } \\
\text { Spoken Danish } \\
\text { Written Danish } \\
\text { Mathematics } \\
\text { Physical education } \\
\text { Total achievement }\end{array}$ & 134 & $\begin{array}{l}1.16(0.44) \\
1.00(0.52) \\
1.10(0.48) \\
1.15(0.52) \\
4.42(2.58)\end{array}$ & 125 & $\begin{array}{l}1.06(0.58) \\
1.04(0.50) \\
1.08(0.45) \\
1.16(0.53) \\
4.37(2 \cdot 15)\end{array}$ & 259 & $\begin{array}{l}1.11(0.48) \\
1.02(0.51) \\
1.09(0.46) \\
1.15(0.52) \\
4.39(2.37)\end{array}$ & $\begin{array}{l}\text { NS } \\
- \\
- \\
-\end{array}$ \\
\hline
\end{tabular}

Six patients with unrelated neurological disorders were excluded (see text). NS=not significant. 
Table 5 Long term outcome after prophylaxis or no prophylaxis; cognitive abilities. Values are mean (SD)

\begin{tabular}{|c|c|c|c|c|}
\hline & $\begin{array}{l}\text { Prophylaxis } \\
(n=91)\end{array}$ & $\begin{array}{l}\text { Control } \\
(n=75)\end{array}$ & $\begin{array}{l}\text { Total } \\
(n=166)\end{array}$ & $\stackrel{p}{\text { Value }}$ \\
\hline \multicolumn{5}{|l|}{ Memory } \\
\hline \multicolumn{5}{|l|}{ Logic (the Goldsmith story) } \\
\hline Meaning, short term recall & $210(60)$ & $207(61)$ & $209(60)$ & NS \\
\hline Meaning, long term recall & $183(61)$ & $184(63)$ & $183(62)$ & - \\
\hline Wording, short term recall & $149(63)$ & $150(59)$ & $149(61)$ & - \\
\hline Wording, long term recall & $105(56)$ & $108(55)$ & $106(55)$ & - \\
\hline \multicolumn{5}{|l|}{ Auditive } \\
\hline \multicolumn{5}{|l|}{ Free recall test } \\
\hline Short term recall & $22 \cdot 5(64)$ & $20 \cdot 3(4 \cdot 2)$ & $21 \cdot 5(5 \cdot 6)$ & 0.03 \\
\hline Long term recall & $44 \cdot 0(5 \cdot 8)$ & $42 \cdot 8(5 \cdot 7)$ & $43.4(5 \cdot 1)$ & NS \\
\hline \multicolumn{5}{|l|}{ Auditive } \\
\hline Digit span, forward & $4.8(1 \cdot 0)$ & $5 \cdot 0(1 \cdot 1)$ & $4.9(1.0)$ & NS \\
\hline Digit span, backward & $3 \cdot 3(1 \cdot 0)$ & $3.1(0.9)$ & $3 \cdot 2(1 \cdot 0)$ & - \\
\hline \multicolumn{5}{|l|}{ Non-verbal $\left(\right.$ BVRT $\left.^{\star}\right)$} \\
\hline Short term recall & $4 \cdot 5(3 \cdot 1)$ & $4 \cdot 1(2 \cdot 4)$ & $4 \cdot 3(2 \cdot 8)$ & NS \\
\hline Motor assessment & $25 \cdot 8(6 \cdot 1)$ & $26.0(5 \cdot 7)$ & $25.9(5.9)$ & - \\
\hline Long term recall & $0.37(0.6)$ & $0.44(0.7)$ & $0.41(0.7)$ & - \\
\hline \multicolumn{5}{|l|}{ Visuo-motor speed } \\
\hline Trail A & $20.5(8.9)$ & $21 \cdot 1(7 \cdot 0)$ & $20 \cdot 8(8 \cdot 1)$ & NS \\
\hline Trail B & $34.4(17 \cdot 8)$ & $36.5(14.6)$ & $35 \cdot 3(11 \cdot 3)$ & - \\
\hline \multicolumn{5}{|l|}{ Continuous visual reaction time test } \\
\hline $50 \%$ centile & $2622(339)$ & $2650(295)$ & $2635(319)$ & NS \\
\hline $10 \%-90 \%$ centile difference & $1065(465)$ & $1057(331)$ & $1061(408)$ & - \\
\hline Total reaction time & $2744(368)$ & $2777(305)$ & $2759(340)$ & - \\
\hline \multicolumn{5}{|l|}{ Reading test } \\
\hline Errors (No) & $3.0(3 \cdot 3)$ & $2 \cdot 6(3.7)$ & $2 \cdot 8(3.5)$ & NS \\
\hline Time (sec) & $15 \cdot 8(5 \cdot 4)$ & $16.8(5.4)$ & $16 \cdot 3(5 \cdot 2)$ & - \\
\hline
\end{tabular}

${ }^{\star}$ BVRT $=$ Benton's revised visual retention test; $\mathrm{NS}=$ not significant.

The IQ results from the general intelligence test (WISC) were also almost identical in the two groups and no significant differences were found for full scale, verbal, or performance IQ (table 4). Furthermore, there were no differences in scaled scores between the two groups on any employed WISC subtests. A significant difference was found on raw scores for the coding subtest with higher scores for the prophylaxis group $(p<0.05)$.

The only significant difference found on the neuropsychological test battery (table 5) was on immediate recall for the free recall test with better recall for the prophylaxis group $(p<0.05)$. The significant differences mentioned probably arose by chance alone. Prior treatment type made no difference in scholastic achievement as evaluated by the teacher at the time of follow up. That was true for both single school subjects and total achievement score (table 4).

Data from the parents' questionnaire showed no differences in long term neurological problems in the two groups (table 6).

Simple and complex febrile convulsions had the same favourable long term outcome in neurological, motor, intellectual, and cognitive ability. This was even the case irrespective of treatment (table 7). The not significant tendency towards better outcome $(0.05<\mathrm{p}<0.1)$ in complex febrile convulsions is unexplained, but probably arose by chance alone or was due to some unidentified selection bias.

\section{Discussion}

This is the first randomised, therapeutical trial in children with febrile convulsions with a long term follow up. Containing two treatment groups with comparable baseline clinical and demographic characteristics, given prophylaxis or not, the design is suitable for addressing the crucial question of whether preventing recurrences influences the long term outcome or not. Our data document that the long term prognosis in terms of occurrence of epilepsy, neurological, motor, intellectual, cognitive, and scholastic attainments is uninfluenced by the type of treatment provided in early childhood as assessed by a broad range of tests and

Table 6 Parents' questionnaire. Long term neurological problems after prophylaxis or no prophylaxis

\begin{tabular}{|c|c|c|c|c|c|c|c|c|c|c|}
\hline & \multicolumn{3}{|c|}{ Prophylaxis } & \multicolumn{3}{|l|}{ Control } & \multicolumn{3}{|l|}{ Total } & \multirow[b]{2}{*}{$\stackrel{p}{\text { Value }}$} \\
\hline & $\begin{array}{l}\text { No with } \\
\text { condition }\end{array}$ & Total & $\%$ & $\begin{array}{l}\text { No with } \\
\text { condition }\end{array}$ & Total & $\%$ & $\begin{array}{l}\text { No with } \\
\text { condition }\end{array}$ & Total & $\%$ & \\
\hline $\begin{array}{l}\text { Epilepsy } \\
\text { Single afebrile seizure } \\
\text { Faintings } \\
\text { Migraine } \\
\text { Tension headache } \\
\text { Reading-writing problems } \\
\text { Motor problems } \\
\text { Special education in school } \\
\text { Febrile convulsions in siblings } \\
\text { Febrile convulsions in parents } \\
\text { Epilepsy in siblings } \\
\text { Epilepsy in parents }\end{array}$ & $\begin{array}{r}2 \\
3 \\
8 \\
8 \\
27 \\
19 \\
12 \\
17 \\
24 \\
13 \\
4 \\
3\end{array}$ & $\begin{array}{l}140 \\
139 \\
140 \\
139 \\
139 \\
140 \\
139 \\
140 \\
139 \\
138 \\
138 \\
128\end{array}$ & $\begin{array}{r}1 \cdot 4 \\
-2 \cdot 2 \\
5 \cdot 7 \\
5 \cdot 8 \\
19 \cdot 4 \\
13 \cdot 6 \\
8 \cdot 6 \\
12 \cdot 1 \\
17 \cdot 2 \\
9 \cdot 4 \\
2 \cdot 9 \\
2 \cdot 2\end{array}$ & $\begin{array}{r}4 \\
2 \\
9 \\
7 \\
25 \\
22 \\
16 \\
16 \\
25 \\
20 \\
6 \\
5\end{array}$ & $\begin{array}{l}128 \\
126 \\
120 \\
129 \\
129 \\
128 \\
113 \\
129 \\
127 \\
127 \\
128 \\
139\end{array}$ & $\begin{array}{r}3 \cdot 1 \\
1 \cdot 6 \\
7 \cdot 0 \\
5 \cdot 4 \\
19 \cdot 4 \\
17 \cdot 2 \\
12 \cdot 4 \\
12 \cdot 4 \\
19 \cdot 7 \\
15 \cdot 7 \\
4 \cdot 7 \\
3 \cdot 9\end{array}$ & $\begin{array}{r}6 \\
5 \\
17 \\
15 \\
52 \\
41 \\
28 \\
33 \\
49 \\
33 \\
10 \\
8\end{array}$ & $\begin{array}{l}268 \\
267 \\
269 \\
253 \\
268 \\
268 \\
240 \\
269 \\
217 \\
232 \\
256 \\
259\end{array}$ & $\begin{array}{r}2.2 \\
1.9 \\
6.3 \\
5.6 \\
19.4 \\
15.3 \\
10.4 \\
12.3 \\
18.4 \\
12.5 \\
3.8 \\
3.0\end{array}$ & $\begin{array}{l}\text { NS } \\
\text { NS } \\
\text { NS } \\
\text { NS } \\
\text { NS } \\
\text { NS } \\
\text { NS } \\
\text { NS } \\
\text { NS } \\
\text { NS } \\
\text { NS } \\
\text { NS }\end{array}$ \\
\hline
\end{tabular}

NS $=$ not significant

Table 7 Long term outcome after simple and complex febrile convulsions given prophylaxis or no prophylaxis; intellectual, scholastic and motor abilities

\begin{tabular}{|c|c|c|c|c|c|c|c|c|c|}
\hline & \multicolumn{4}{|c|}{ Simple febrile convulsions } & \multicolumn{4}{|c|}{ Complex febrile convulsions } & \multirow[b]{3}{*}{$\stackrel{p}{\text { Value }}$} \\
\hline & \multicolumn{2}{|c|}{ Prophylaxis } & \multicolumn{2}{|l|}{ Controls } & \multicolumn{2}{|c|}{ Prophylaxis } & \multicolumn{2}{|l|}{ Controls } & \\
\hline & $\begin{array}{l}\text { No of } \\
\text { children }\end{array}$ & Mean $(S D)$ & $\begin{array}{l}\text { No of } \\
\text { children }\end{array}$ & Mean $(S D)$ & $\begin{array}{l}\text { No of } \\
\text { children }\end{array}$ & Mean (SD) & $\begin{array}{l}\text { No of } \\
\text { children }\end{array}$ & Mean (SD) & \\
\hline WISC & 72 & & 57 & & 14 & & 15 & & \\
\hline $\begin{array}{l}\text { Verbal IQ } \\
\text { Performance IQ } \\
\text { Full scale IQ }\end{array}$ & & $\begin{array}{l}104(16) \\
113(14) \\
109(13)\end{array}$ & & $\begin{array}{l}104(16) \\
111(19) \\
107(15)\end{array}$ & & $\begin{array}{l}110(16) \\
120(12) \\
115(12)\end{array}$ & & $\begin{array}{l}110(16) \\
111(22) \\
111(17)\end{array}$ & $\begin{array}{l}\text { NS } \\
\text { NS } \\
\text { NS }\end{array}$ \\
\hline Scholastic achievement & & & & & & & & & \\
\hline $\begin{array}{l}\text { (school score) } \\
\text { Stott motor test }\end{array}$ & $\begin{array}{r}105 \\
73\end{array}$ & $4 \cdot 4(2 \cdot 5)$ & $\begin{array}{l}96 \\
59\end{array}$ & $4 \cdot 2(2 \cdot 1)$ & $\begin{array}{l}17 \\
14\end{array}$ & $5 \cdot 7(2 \cdot 4)$ & $\begin{array}{l}22 \\
15\end{array}$ & $5 \cdot 1(2 \cdot 3)$ & NS \\
\hline $\begin{array}{l}\text { Fine motor } \\
\text { Gross motor } \\
\text { Total motor }\end{array}$ & & $\begin{array}{l}1.4(1 \cdot 5) \\
3 \cdot 1(3 \cdot 5) \\
4 \cdot 5(4 \cdot 6)\end{array}$ & & $\begin{array}{l}1.6(1.6) \\
3.6(3.4) \\
5 \cdot 2(4 \cdot 7)\end{array}$ & & $\begin{array}{l}1 \cdot 0(1 \cdot 5) \\
2 \cdot 6(2 \cdot 3) \\
3 \cdot 6(2 \cdot 9)\end{array}$ & & $\begin{array}{l}1 \cdot 5(1 \cdot 7) \\
3 \cdot 1(4 \cdot 0) \\
4 \cdot 1(5 \cdot 6)\end{array}$ & $\begin{array}{l}\text { NS } \\
\text { NS } \\
\text { NS }\end{array}$ \\
\hline
\end{tabular}

NS $=$ not significant 
from the viewpoint of paediatricians, neuropsychologists, teachers, and the family itself. Even though the applied prophylaxis effectively reduced the 18 month recurrence rate in early childhood $^{14}$ we were unable to document any long term therapeutic gain compared with acute treatment of ongoing convulsions. This includes the long term risk of epilepsy, which was very low $(0 \cdot 7 \%)$ and almost identical whether prior prophylaxis $(0.7 \%)$ or acute anticonvulsant treatment only $(0.8 \%)$ had been given. It has long been discussed whether febrile convulsions may presage neurological, motor, intellectual, and cognitive dysfunction. Our encouraging findings are in accordance with recent epidemiological data from major cohorts $^{1-7}$ and all available evidence, except for the Danish twin study ${ }^{13}$ and retrospective data from highly selected neurosurgical cases ${ }^{17} 18$ or other highly specialised referral centres, ${ }^{19}$ strongly suggests a favourable long term outcome. The mean WISC IQ score in our series (full scale $I Q=109$ ) was quite similar to the IQs found in Danish schoolchildren not suffering from febrile convulsions (full scale $\mathrm{IQ}=$ 110) (personal communication), suggesting a fully benign outcome. However, it should be stressed that by comparing two therapeutical strategies our data may not be meaningful for untreated children.

More surprising are the findings that simple and complex febrile convulsions had similar long term prognosis in all aspects of neurological, motor, intellectual, cognitive, and scholastic achievements. Not even a trend towards dysfunction was seen after complex febrile convulsions, felt by many to have potentially serious consequences for the child. The favourable outcome in complex febrile convulsions in our series may well reflect the efficiency of the primary health care system, short transit time to hospital and advances in treatment, including supportive care techniques and an aggressive anticonvulsant treatment of ongoing seizures. Our results are in accordance with those of two major cohorts. 1256 Our study also showed that children with complex febrile convulsions put on prophylaxis did not fare any better in the long run than when given acute anticonvulsant treatment, suggesting that similar therapeutic principles could be applied to both types. However, the subgroups were small making data less reliable, and stratification of the data into multiple, prolonged, or focal febrile convulsions was not possible.

The study emphasises that prevention of epilepsy is not a realistic target of prophylaxis, as the long term occurrence of epilepsy was low $(0 \cdot 7 \%)$ even after complex febrile convulsions $(2.5 \%)$ and uninfluenced by type of treatment. All cases were classified as generalised idiopathic or localisation related epilepsies, usually considered to be of genetic origin. It is impressive that not even a single case of temporal lobe epilepsy caused by underlying hippocampal sclerosis has emerged after 12 years of observation among some 300 children with prior febrile convulsions, confirming that this sequence of events appears to be rare. ${ }^{6}$
In conclusion, preventing new febrile convulsions appears no better in the long run than abbreviating them. Therapeutic suggestions should take into account that these largely benign convulsions are extremely upsetting for the parents and that long lasting recurrences or febrile status with a less benign outcome in rare cases $^{620}$ do occur unpredictably. It seems reasonable to recommend that all families with children suffering from febrile convulsions be equipped with one or two doses of rectal diazepam in solution ${ }^{21-24}$ or other rapidly acting benzodiazepines 2526 for abbreviating new febrile convulsions, especially the long lasting, potentially central nervous system damaging variety. Intermittent, short term prophylaxis with benzodiazepines given at times of fever, which effectively reduces the recurrence rate, ${ }^{10-1427-37}$ should probably be reserved for a few selected cases. These less well defined cases may tentatively comprise those with many or long lasting recurrences or many risk factors for further febrile fits. ${ }^{15} 3839$ The treatment is not totally devoid of side effects, but our study also showed that the administration of benzodiazepines to young children at times of fever or in the acute situation had no detrimental effect on their later neurological, motor, intellectual, cognitive, or scholastic achievement.

This study was supported by grants from the Danish Medical Research Counsil (12-0238-1), Queen Louise's Children Hospital grant, the Danish Epilepsy Society and Sygekassernes Helsefond.

We thank Michael Davidsen, Herlev University Hospital, for performing the data processing and statistical analysis.

1 Nelson KB, Ellenberg JH. Predictors of epilepsy in children who have experienced febrile seizures. N Engl f Med 1976; 295: 1029-33.

2 Nelson KB, Ellenberg JH. Prognosis in children with febrile seizures. Pediatrics 1978; 61: 720-7.

3 Nelson KB, Ellenberg JH. Febrile seizures. New York: Raven Press, 1981.

4 Roos EM, Peckham CS, West PB, Butler NR. Epilepsy in childhood: findings from the national child development study. $B M \mathcal{F}$ 1980; 280: 207-10.

5 Verity CM, Butler NR, Colding J. Febrile convulsions in a national cohort followed up from birth. II. Medical history and intellectual ability at 5 years of age. $B M \mathcal{F} 1985 ; 290$ : 1311-5.

6 Verity CM, Golding J. Risk of epilepsy after febrile convulsions: a national cohort study. BMF 1991; 303: 1373-6.

7 Annegers JF, Hauser WA, Shirts SB, Kurland LT. Factors prognostic of unprovoked seizures after febrile convulprognostic of unprovoked seizures af
sions. N Engl f Med 1987; 316: 493-8.

8 Knudsen FU. Optimum management of febrile seizures in childhood. Drugs 1988; 36: 111-20.

9 Newton RW, McKinlay I. Subsequent management of children with febrile convulsions. Dev Med Child Neurol 1988; 30: 402-6.

10 Knudsen FU. Intermittent diazepam prophylaxis in febrile convulsions. Acta Neurol Scand 1991; 83 (suppl): $1-24$.

11 Research Unit of Royal College of Physicians and British Paediatric Association, London. Guidelines for the management of convulsions with fever. BMF 1991; 303: 634-6.

12 Wallace SJ. The child with febrile seizures. London: Butterworth, 1988

13 Schiøttz-Christensen E. Feberkramper. Studier af atiologi og folgetilstande med udgangspunkt $i$ tvillingeundersegelser. folgetilstande med udgangspunkt ${ }^{i}$

14 Knudsen FU. Effective short-term diazepam prophylaxis in febrile convulsions. F Pediatr 1985; 106: 487-90.

15 Knudsen FU. Recurrence risk after first febrile seizure and effect of short term diazepam prophylaxis. Arch Dis Child 1985; 60: 1045-9.

16 Whiting HT, Clarke TA, Morris PR. A clinical validation of the Stott test of motor impairment. British fournal of Social and Clinical Psychology 1969; 8: 270-4.

17 Taylor DC, Ounsted C. Biological mechanisms influencing the outcome of febrile seizures in response to fever. Epilepsy 1971; 12: 33-45.

18 Falconer MA. Mesial temporal sclerosis as a common cause of epilepsy: aetiology, treatment and prevention. Lancet 1974; ii: 767-70

19 Aicardi J, Chevrie JJ. Convulsive status epilepticus in infants 
and children; a study of 239 cases. Epilepsia 1971; 11: 187-97.

20 Verity CM, Ross EM, Golding J. Outcome of childhood status epilepticus and lengthy febrile convulsions: findings status epilepticus and lengthy febrile convulsions:
of national cohort study. $B M F$ 1993; 367: 225-8.

21 Knudsen FU. Plasma-diazepam in infants after rectal administration in solution and by suppository. Acta Paediatr Scand 1977; 66: 563-7.

22 Knudsen FU. Rectal administration of diazepam in solution in the acute treatment of convulsions in infants and children. Arch Dis Child 1979; 54: 855-7.

23 Hoppu K, Santavuori P. Diazepam rectal solution for home treatment of acute seizures in children. Acta Paediatr Scand 1981; 70: 369-72.

24 Ventura A, Basso T, Bortolan G, et al. Home treatment of seizures as a strategy for the long term management of febrile convulsions in children. Helvetica Paediatrica Acta 1982; 37: 581-7.

25 Rylance GW, Poulton J, Cherry RC, Cullen RE. Plasma concentrations of clonazepam after single rectal administration. Arch Dis Child 1986; 61: 186-8.

26 Dooley JM, Tibbles JAR, Rumney PG, Dooley KC. Recta lorazepam in the treatment of acute seizures in childhood. Ann Neurol 1985; 18: 412-3.

27 Knudsen FU, Vestermark S. Prophylactic diazepam or phenobarbitone in febrile convulsions: a prospective controlled study. Arch Dis Child 1978; 53: 660-3.

28 Thorn I. Prevention of recurrent febrile seizures: intermittent prophylaxis with diazepam compared with continuous treatment with phenobarbital. In: Nelson KB Ellenberg JH, eds. Febrile seizures. New York: Raven Press, 1981: 119-26.

29 Echenne B, Cheminal R, Martin P, et al. Utilisation du diazépam dans le traitement préventif à domicile des récidives de convulsions fébriles. Arch Fr Pediatr 1983; 40: 499-501.

30 Garcia FO, Campos-Castello J, Maldonado JC. Fenobarbital oral continuado o diazepam rectal intermitente para la prevención de la crisis febriles. An Esp mitente para la prevencion
Pediatr 1984; 20: 763-9.

31 Lee K, Taudorf K, Hvorslev V. Prophylactic treatment with valproic acid or diazepam in children with febrile convulvalproic acid or diazepam in children with $\mathrm{f}$
sions. Acta Paediatr Scand 1986; 75: 593-7.

32 Mosquera C, Rodrigues J, Cabrero A, Fidalgo I, Fernande RM. Prevención de la recurrencia de crisis febriles profilaxis intermitente con diazepam rectal comparada in tratamiento continuo im valproato sódica. An Esp Pediat 1987; 27: 379-81.

33 Autret E, Billard C, Bertrand P, Motte J, Pouplard F, Jonville AP. Double-blind, randomized trial of diazepam versus placebo for prevention of recurrence of febrile seizures. F Pediatr 1990; 117: 490-4.

34 Shirai H, Miura H, Sunaoshi W. A clinical study on the effectiveness of intermittent therapy with rectal diazepam suppositories for prevention of recurrent febrile convulsions. A further study. fournal of the fapanese Epilepsy sions. A further study.
Society 1988; 6: 1-10.

35 Ramakrishnan K, Thomas K. Long term prophylaxis of febrile seizures. Indian f Pediatr 1986; 53: 397-400.

36 Vanasse M, Masson P, Geoffroy G, Larbrisseaú A, David PC. Intermittent treatment of febrile convulsions with nitrazepam. Can $\mathcal{f}$ Neurol Sci 1984; 11: 377-9.

37 Rosman N, Colton T, Labazzo J, et al. A controlled trial of diazepam administered during febrile illnesses to preven recurrence of febrile seizures. $N$ Engl $\mathcal{F}$ Med 1993; 329 79-84.

38 Berg AT, Shinnar S, Hauser WA, Leventhal JM. Predictor of recurrent febrile seizures: a metaanalytic review. $\mathcal{F}$ Pediatr 1990; 116: 329-37.

39 Offringa M, Bossuyt PMM, Lobsen J, et al. Rick factors for seizure recurrence in children with febrile seizures. pooled analysis of individual patient data from five studies. $\mathcal{f}$ Pediatr 1993; 124: 574-84.

\section{Appendix \\ MEMORY FUNCTIONS}

Memory for stories (Binet: the Goldsmith story)

The test measures short and long term memory for meaningful material. A short story is read to the subject and immediately afterwards the story has to be repeated as literally as possible. Retention is measured one hour after the initial presentation. Both immediate and long term recall is scored according to how well the important parts of the story are remembered and how well the exact wording of the story is maintained.

Digit span, forward and backward

This test is similar to the WISC subtest digit span, but the subject gets two sequences for each number of digits and the score is the total number of sequences recalled correctly. Digit span forward and backward are also scored separately.

\section{Free recall test}

The test measures short term memory and retention for unrelated material. The test material comprises lists of 15 monosyllabic nouns (six different lists). The lists are read aloud once at the rate of one word per second, and the subject has to recall the words he remembers. The retention task is designed as a two alternatives, forced choice procedure. Performance is scored with respect to total number of nouns recalled immediately after presentation and total numbers of recognised nouns after a 10 minutes delay.

\section{Benton's revised visual retention test (BVRT)}

The test measures visual perception and visual short term memory. The test material comprises 10 pictures of one to three rather complex geometrical designs. Each picture is presented for 10 seconds (administration $A$ ) and the subject is required to draw it immediately afterwards. The score is the total number of errors for all 10 pictures.

Long term retention for designs - To get information about visual long term memory for designs a three alternatives, forced choice recognition test was constructed. The pictures shown during administration $\mathrm{A}$ was mixed with designs from parallel series from the BVRT. Recognition is measured after an interval of 10 minutes. Scoring is the total number of correctly recognised designs.

\section{VISUOMOTOR FUNCTIONS}

\section{The Trail tests}

These are so-called tracking tests and measure the rate of complex psychomotoric functioning. Trail $A$ : The subject is required, under time pressure, to connect the numbers 1 to 25 placed in random order on a piece of paper. Trail B: The requirements are essentially similar to those of trail A, except that the subject must alternate between numeric and alphabetic series. The score in trail tests is the number of seconds required to finish the task.

\section{Continuous visual reaction time test}

The test measures hand-eye coordination and the ability to sustain focused attention. The subject is seated in front of a computer. At random intervals a white square is shown on the screen, and the subject is required to make the square disappear as fast as possible by pressing the space bar. Total reaction time is recorded in centiseconds, and the scores for centiles $10,50,90$, the difference between centiles 90 and 10, and total reaction time is computed.

To get an evaluation of fine motor ability the drawings from the BVRT were scored with regard to motor execution. 